\title{
PREDICTION OF BENEFIT FROM CONSOLIDATION CHEMOTERAPY FOR CERVICAL CANCER PATIENTS USING A CLINICAL PROGNOSTIC SCORE
}

A. Santos ${ }^{1}$, V. Fabri1 ${ }^{1}$, A. Queiroz ${ }^{1}$, H. Mantoan ${ }^{2}$, S. Sanches ${ }^{1}$, A. Guimarães ${ }^{1}$, A. Ribeiro ${ }^{1}$, R. Souza ${ }^{1}$, J. Maya ${ }^{1}$, E. Santos ${ }^{1}$, F. Castro ${ }^{1}$, L. João Paulo ${ }^{1}$, M. $\mathrm{Chen}^{3}$, G. Baiocchi ${ }^{2}$, A. Da Costa ${ }^{1} . \quad{ }^{1}$ Medical Oncology ${ }^{2}$ Gynecological Oncology ${ }^{3}$ Radiotherapy, São Paulo, Brazil

Objectives: The use of consolidation chemotherapy (CCT) after chemoradiation (CRT) in cervical cancer remains debatable. We evaluated the impact of CCT added to standard CRT and sought to identify predictive factors of CCT benefit.

Methods: This retrospective study reviewed 216 patients with 2014 FIGO stage IB2-IIA2, and IIB-IVB (para-aortic nodes only) cervical cancer treated with CRT alone or CRT followed by CCT (CCT group). Firstly, we assessed the prognostic role of CCT. Moreover, we developed a prognostic score for distant metastasis free survival (DMFS).

\begin{tabular}{|c|c|c|c|}
\hline \multirow[t]{2}{*}{ Characteristics } & \multicolumn{2}{|c|}{ Freq (\%) } & \multirow[t]{2}{*}{$\mathbf{p}$} \\
\hline & Consolidation CT & No Consolidation CT & \\
\hline Number of patients (\%) & $72(33.3)$ & $144(66.7)$ & \\
\hline Age (median/P25-75) & $40(34-53)$ & $50(42-63)$ & \\
\hline$\leq 35$ & $21(29.2)$ & 15 (10.4) & $<0.001$ \\
\hline$>35$ & $51(70.8)$ & $144(89.6)$ & \\
\hline \multicolumn{4}{|l|}{ RT 2D } \\
\hline No & $67(93.1)$ & $26(18.6)$ & $<0.001$ \\
\hline Yes & $5(6.9)$ & $114(81.4)$ & \\
\hline \multicolumn{4}{|l|}{ ECOG } \\
\hline 0 & $44(61.1)$ & $90(63.8)$ & 0.698 \\
\hline$\geq 1$ & $28(38.9)$ & $51(36.2)$ & \\
\hline \multicolumn{4}{|l|}{ Histology } \\
\hline CEC & $52(72.2)$ & $110(78.6)$ & 0.302 \\
\hline Adeno & $20(28.1)$ & 30 (21.4) & \\
\hline \multicolumn{4}{|l|}{ Grade } \\
\hline$\leq 2$ & $40(69.0)$ & $66(58.4)$ & 0.178 \\
\hline 3 & $18(31.0)$ & 47 (41.6) & \\
\hline \multicolumn{4}{|l|}{ Size } \\
\hline$>4 \mathrm{~cm}$ & $8(15.7)$ & $19(21.3)$ & 0.414 \\
\hline$\geq 4 \mathrm{~cm}$ & $43(84.3)$ & $70(78.7)$ & \\
\hline \multicolumn{4}{|l|}{ Stage } \\
\hline$\leq I I B$ & $6(8.3)$ & $16(11.3)$ & 0.494 \\
\hline$\geq I I I A$ & 66 (91.7) & $125(88.7)$ & \\
\hline \multicolumn{4}{|l|}{ Lymphnode } \\
\hline positive & $28(38.9)$ & $61(45.2)$ & 0.383 \\
\hline Negative & $44(61.1)$ & $74(54.8)$ & \\
\hline \multicolumn{4}{|l|}{ Concomitant CT } \\
\hline$<6$ cycles & $17(23.6)$ & $47(35.6)$ & 0.078 \\
\hline$\geq 6$ cycles & 55 (76.4) & $85(64.4)$ & \\
\hline \multicolumn{4}{|l|}{ Brachytherapy } \\
\hline Yes & $69(95.8)$ & $124(87.9)$ & 0.062 \\
\hline No & $3(4.2)$ & $17(12.1)$ & \\
\hline
\end{tabular}

Table 1 - Clinical characteristics

\begin{tabular}{|c|c|c|c|c|c|c|}
\hline \multirow{2}{*}{ Characteristic } & \multicolumn{2}{|l|}{ OS } & \multicolumn{2}{|l|}{ PFS } & \multicolumn{2}{|l|}{ DMFS } \\
\hline & HR (95\% Cl) & p & HR (95\% Cl) & $\mathbf{p}$ & HR (95\% Cl) & $\mathbf{p}$ \\
\hline \multicolumn{7}{|l|}{ Consolidation CT } \\
\hline No & 1 & 0.023 & 1 & 0.005 & 1 & 0.010 \\
\hline Yes & $0.35(0.15-0.87)$ & & $0.41(0.22-0.76)$ & & $0.40(012-0.80)$ & \\
\hline \multicolumn{7}{|l|}{ Stage } \\
\hline$\leq \| \mathrm{IB}$ & 1 & 0.037 & 1 & 0.014 & 1 & 0.06 \\
\hline$\geq I I I A$ & $2.00(1.04-3.86)$ & & $2.03(1.15-3.57)$ & & $1.86(0.99-3.49)$ & \\
\hline \multicolumn{7}{|l|}{ Concomitant CT } \\
\hline$\geq 6$ cycles & 1 & 0.019 & 1 & 0.001 & 1 & 0.03 \\
\hline$<6$ cycles & $2.13(1.14-4.00)$ & & $2.04(1.19-3.50)$ & & $1.94(1.06-3.56)$ & \\
\hline \multicolumn{7}{|l|}{ RT 2D } \\
\hline No & 1 & 0.236 & - & & - & \\
\hline Yes & $1.52(0.76-3.04)$ & & & & & \\
\hline \multicolumn{7}{|l|}{ Histology } \\
\hline CEC & - & & 1 & $<0.001$ & 1 & $<0.001$ \\
\hline Adeno & & & $3.07(1.66-5.66)$ & & $3.52(1.77-7.00)$ & \\
\hline \multicolumn{7}{|l|}{ Lymphnode } \\
\hline Negative & - & & 1 & 0.002 & 1 & $<0.001$ \\
\hline Positive & & & $2.60(1.41-4.88)$ & & $3.85(1.84-8.05)$ & \\
\hline \multicolumn{7}{|l|}{ Grade } \\
\hline$\leq 2$ & - & & - & & - & \\
\hline 3 & & & & & & \\
\hline
\end{tabular}

Table 2 - Multivariable cox regression analysis for OS, PFS, DMFS and LRFS
Results: After 42.8 months of median follow up 144 patients were treated with standard CRT and 72 with CCT. Clinical characteristics were comparable between groups, except CCT patients were younger $(p<0.001)$ and less frequently treated with $2 \mathrm{D}$ radiation techniques $(81.4 \%$ vs. $93.1 \%, \mathrm{p}=0.023)$ (table 1). Median survivals were not reached in both groups. In multivariate analyses, CCT was related to longer overall survival (OS) (HR 0.35, $\mathrm{p}=0.023)$, progression free survival (HR 0.41, $\mathrm{p}=0.005$ ) and DMFS (HR 0.40, $\mathrm{p}=0.010$ ) but not locoregional control (table 2)(figure $1 \mathrm{~A}$ and $\mathrm{B}$ ). Potential negative factors for DMFS included lymph node status, adenocarcinoma histology, and stage III or IV and formed a four-tier score (0 to 3 points) with good discrimination $(\mathrm{p}<0.001)$ (figure $1 \mathrm{C})$. The benefit of CCT was present among patients with a score $>1$ (OS:p=0.014; DMFS: $p=0.023$ ) but not for patients with score $\leq 1$ (OS: $p=0.310$; DMFS: $p=0.179$ ) (figure 2$)$..

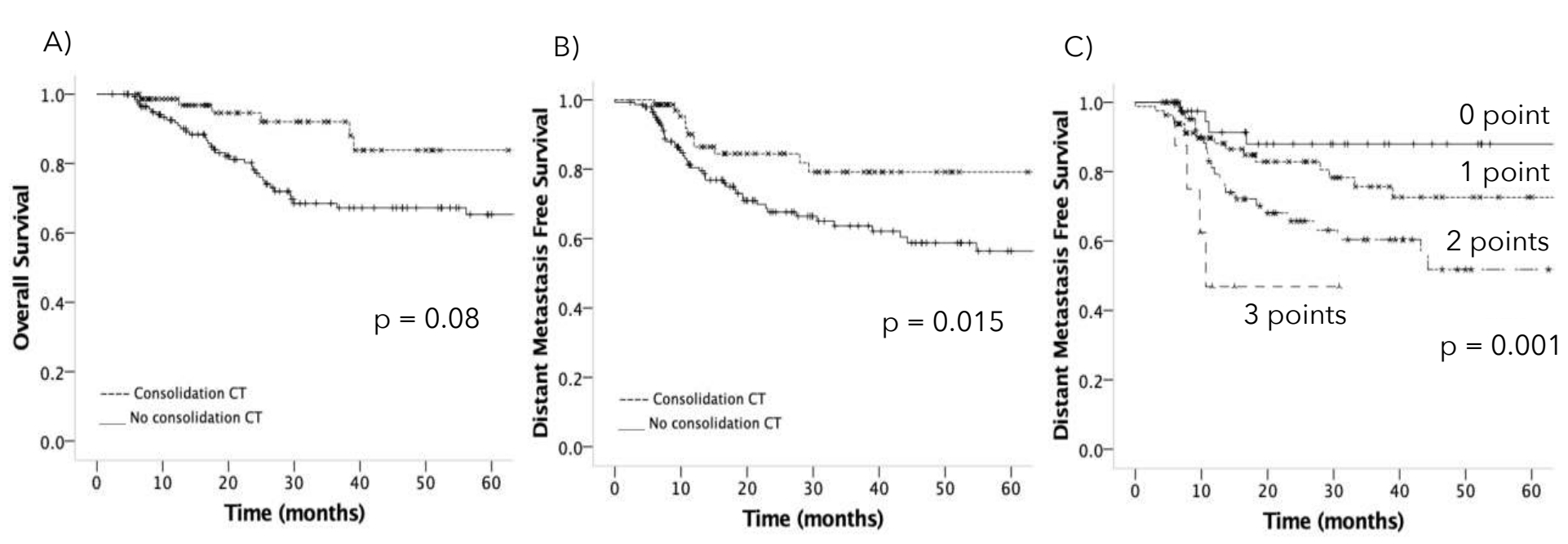

Figure 1 - Overall survival (A) and distant metastasis free survival (B) according to consolidation chemotherapy. (C) Distant metastasis free survival according to the four tier prognostic score

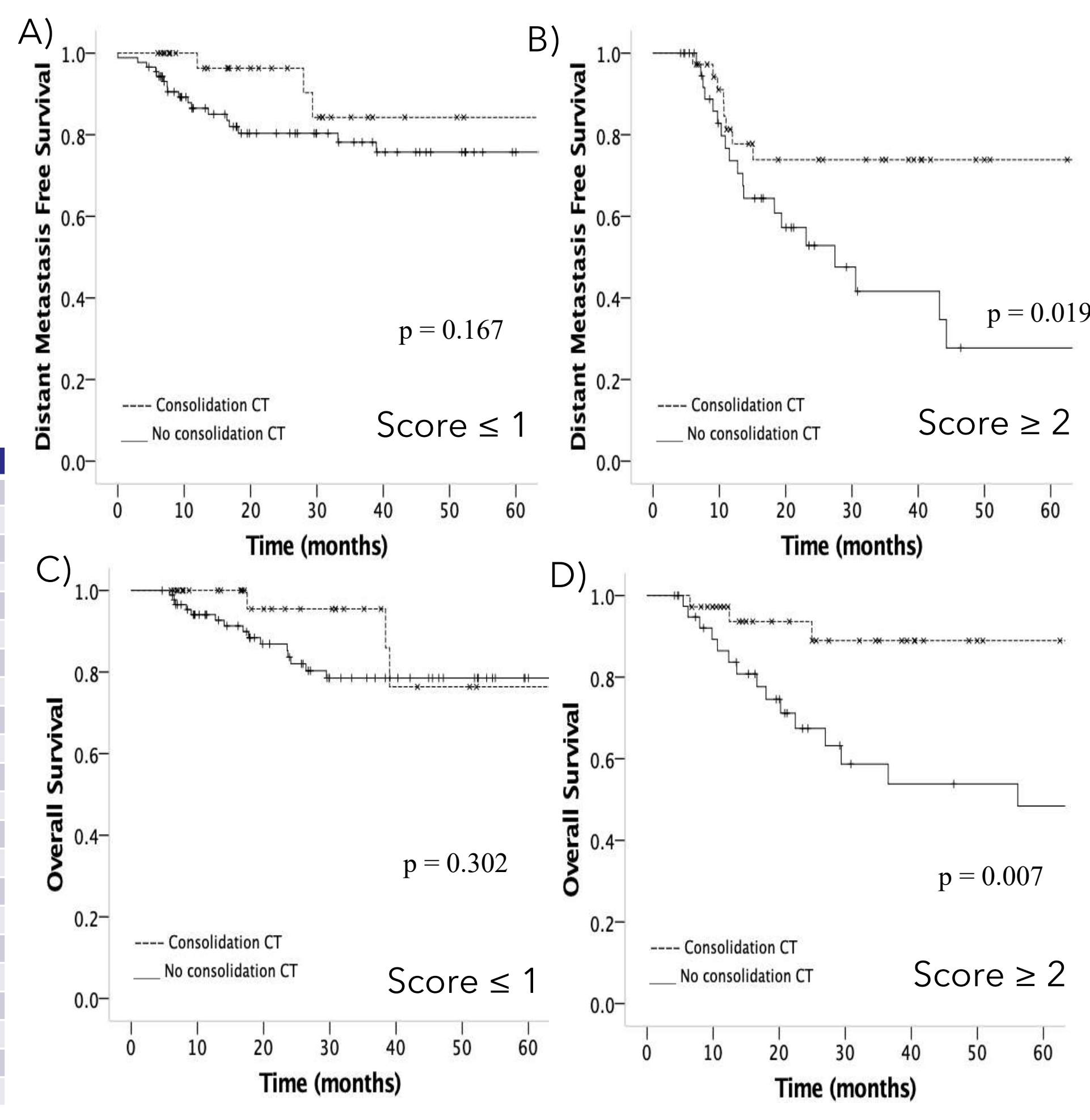

Figure 2 - Distant metastasis free survival (DMFS) according to consolidation chemotherapy in the subgroups of patients with score $\leq 1$ (A) or score $\geq 2$ (B). Overall survival (OS) according to consolidation chemotherapy in the subgroups of patients with score $\leq 1$ (C) or score $\geq 2$ (D). 